\title{
Experimental and finite element analysis of superelastic behaviour of shape memory alloy for damping applications
}

\author{
F. Thiebaud ${ }^{1,2, a}$ And T. Ben Zineb ${ }^{1,2}$ \\ 1 Université de Lorraine, LEMTA UMR 7563, 54500 Vandoeuvre-les-Nancy, France \\ 2 CNRS, LEMTA UMR 7563, 54500 Vandoeuvre-les-Nancy, France
}

Received 17 June 2013, Accepted 14 May 2014

\begin{abstract}
Shape memory alloys (SMA) are good candidates especially for being used as passive dampers. In order to develop the use of these alloys in structural vibrations control, the dynamical behavior of a NiTi helical spring is led, and the damping effect investigated. First, compression tests on the spring are carried out. These tests allow us to notice the effect of the maximal compression displacement, the cyclic behavior and the compression rate on its mechanical behavior. A finite element model analysis of the compression tests is then proposed. In consequence, the materials parameters have been identified after a numerical convergence test. In order to characterize the dynamical behavior of the spring, the innovative tool called equivalent complex stiffness is developed and used. Finally, the one degree of freedom vibration equation is solved with this equivalent complex stiffness. The solution of this equation clearly shows the non linear dynamical behavior of the SMA spring and its damping potential.
\end{abstract}

Key words: Shape memory alloys / experimental compression test / phenomenological model / finite element analysis / equivalent complex stiffness / dynamical behavior / damping

\section{Introduction}

Shape memory alloys (SMAs) are widely studied as smart materials because of their potentiality to be used as dampers, absorbers or actuators elements. For damping applications, an understanding of the material dynamic behavior is needed. One uses the loss of stiffness linked to the martensite transformation between the mother phase called austenite (A) and the product phase called martensite (M). In this case, the SMA elements are used as absorbers mainly for seismic applications [1-5]. In the present paper, the damping effect of a superelastic NiTi helical spring in compression is investigated. One notices that the study of such device has been carried out in previous investigations, experimentally by Speicher et al. [6], numerically by Mirzaeifar [7]. Nevertheless, a lack of results on the damping effect is still present. Thus, an innovative model of the damping effect is proposed in this paper. To do this, in a first step, an experimental compression test is led. In a second step, a three dimensional (3D) model describing the thermo-mechanical behavior of SMAs and implemented in ABAQUS ${ }^{\circledR}$ is used to simulate the compression cycles. A comparison between

\footnotetext{
a Corresponding author:

frederic.thiebaud@univ-lorraine.fr
}

the experimental and the numerical investigations is done in order to validate the $3 \mathrm{D}$ model implementation. In the third part, the equivalent complex stiffness is described and the damping evolutions are investigated. Finally, the dynamic behavior of the helical spring is quickly carried out with the Bode diagrams.

\section{Experimental investigations}

\subsection{Experimental protocol}

By considering the geometrical parameters of the SMA helical spring on Figure 1a, a specific compression tool designed for the spring was adapted on a $100 \mathrm{kN}$ Zwick-Roell ${ }^{\circledR}$ tensile test machine shown on Figure 1b. In order to reduce the friction phenomenon between the spring and the tool, vaseline oil is used as well.

This specific tool allows the test machine to be used in tensile configuration with maximum of safety for the operator. The tests performed are as following: monotonic compression tests with various load levels, cyclic effects and loading rates are also considered. The spring was made from Nitinol Alloy 508 (50.8\% at.\% Nickel) by heating small sequential sections of the stock with a small torch just to the point of initial softening. The softened metal was then bent around a mandrel to produce a helix. 


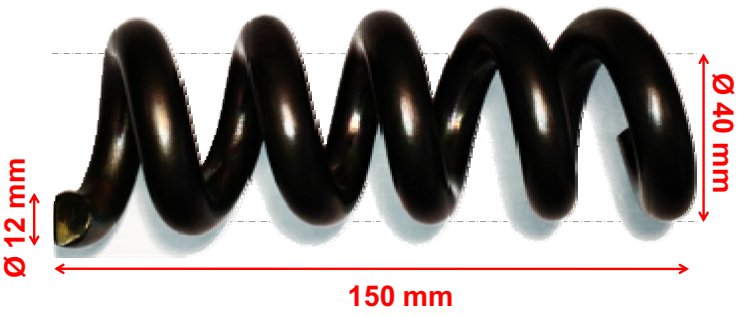

(a)

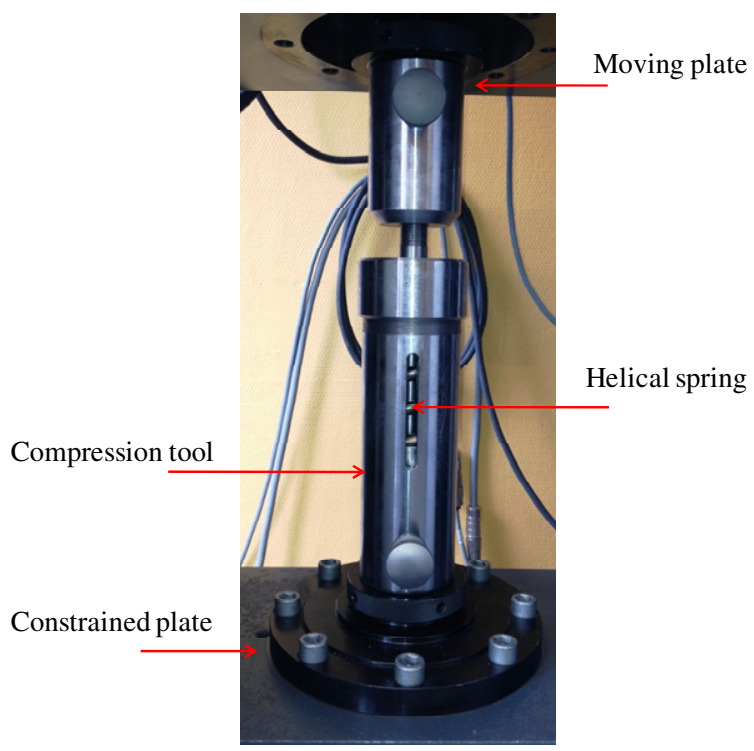

(b)

Fig. 1. Design of the helical spring (a) and experimental compression tool (b).

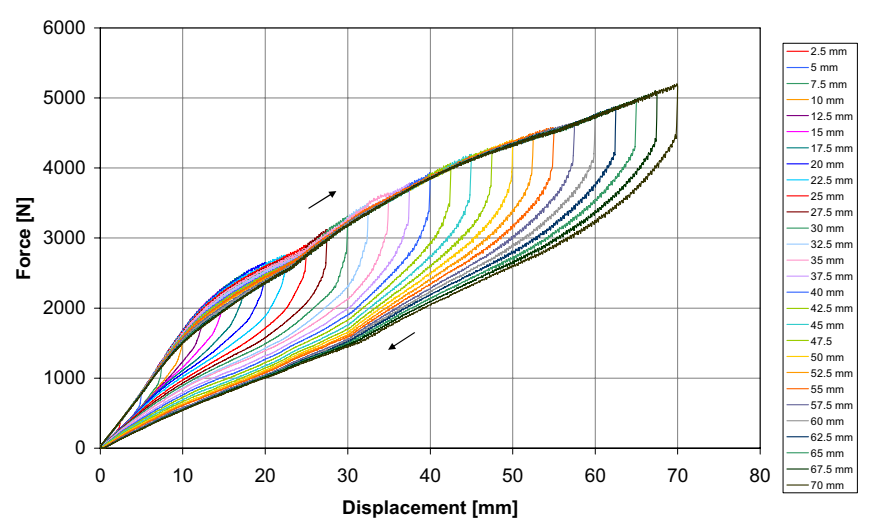

Fig. 2. Resulting force versus the displacement of the moving plate for different displacement levels.

The finished coils were given, a final heat treatment was carried out to achieve uniform properties and good superelasticity.

\subsection{Compression tests}

The evolution of the resulting compression force versus the displacement of the moving plate is given on the Figure 2. A quasi-static loading rate for the testing was set at $2.5 \mathrm{~mm} / \mathrm{s}$ to eliminate dynamic (thermomechanical coupling) effects. Indeed, at this loading rate, the spring can easily dissipate the heat due to the thermomechanical coupling. Additionally, all experiments were carried out under ambient temperature in the range of $21-23{ }^{\circ} \mathrm{C}$.

The typical non linear behavior with mechanical hysteresis of SMA is shown on theses curves. This non linear behavior with hysteresis is explained by the transformation phase $(\mathrm{A} \leftrightarrow \mathrm{M})$ which operates where the local stress is upper than the beginning forward (reverse) transformation stress. Consequently, this device appears to be a good passive damper.

\subsection{Influence of the number of cycles and the loading rate}

Thirty cycles for three different maximum displacements $(17.5,30$ and $57.5 \mathrm{~mm})$ are shown on the Figure $3 \mathrm{a}$. These curves clearly show that the number of cycles does not affect the mechanical behavior of the helical spring. Furthermore, three different loading rates $(0.5,5$ and $10 \mathrm{~mm} / \mathrm{s}, f \approx 0.3 \mathrm{~Hz}$ ) for the same three maximum displacements are tested. These curves highlight that the loading rate has no influence on the mechanical behavior too. It is known that the forward transformation $(\mathrm{A} \rightarrow \mathrm{M})$ is exothermal and the reverse transformation $(\mathrm{M} \rightarrow \mathrm{A})$ endothermal. It is a structure effect: the spring due to its important exchange area with the surrounding air evacuates the heat for this loadings range (convection effect) very easily. This phenomenon can be studied with a heat camera for example and will be done in a future work.

\section{Numerical investigations}

\subsection{A thermomechanical model for the superelasticity}

\subsubsection{Statement of thermomechanical dynamical problem}

Under the assumptions of small strain and displacements, the thermomechanical problem satisfies the following fundamental equations:

- The mechanical equilibrium :

$$
\rho \ddot{\vec{u}}-\vec{\nabla} \cdot \underline{\sigma}=\vec{f}
$$




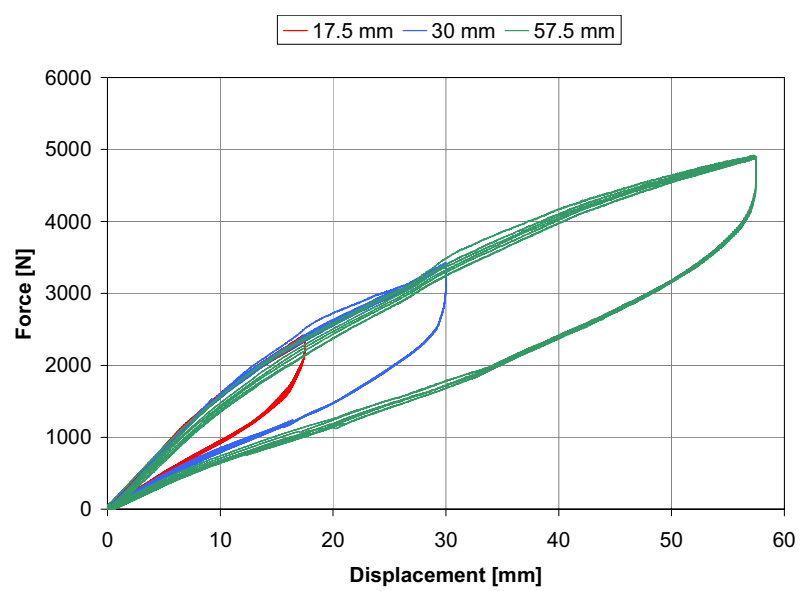

(a)

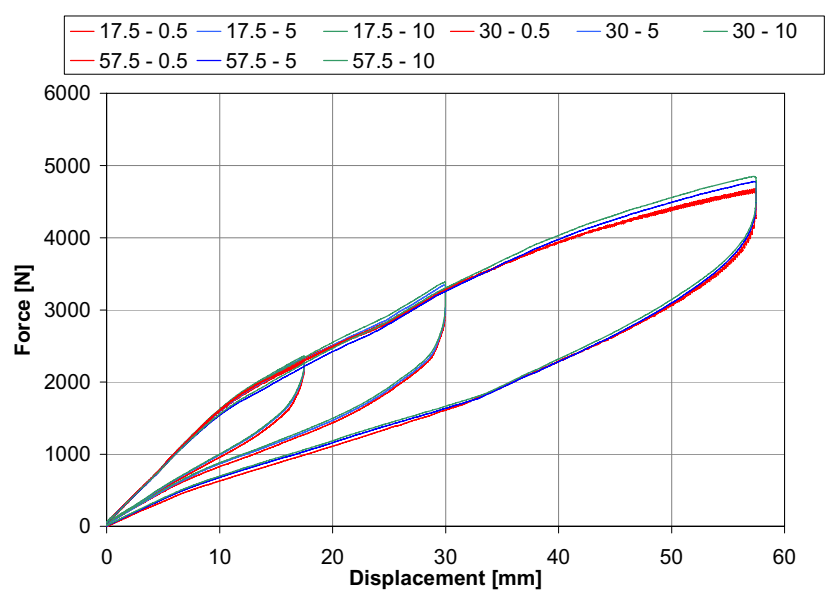

(b)

Fig. 3. Influence of the number of cycles (a) and the loading rate (b).

where $\rho$ represents the material density and $\underline{\sigma}$ the Cauchy stress tensor;

- The first thermodynamic principle (energy conservation without source term):

$$
\rho \dot{e}-\vec{\nabla} \cdot \vec{q}=\underline{\sigma}: \underline{\dot{\varepsilon}}+r
$$

where $e$ is the total energy, $\vec{q}$ the vector of thermal flux, $r$ the internal heat source and $\underline{\varepsilon}$ the Green-Lagrange strain sensor;

- The second thermodynamic principle:

$$
\rho T \dot{s}-\rho \cdot r+\vec{\nabla} \cdot \vec{q}-\frac{1}{T} \vec{q} \cdot \vec{\nabla} T \geqslant 0
$$

where $s$ represents the specific entropy of the material.

\subsubsection{Shape memory alloy behavior}

The behavior considered in this paper is related to the phase change of SMA. At stress-free state, the material is assumed to be fully austenitic. During loading, the phase can change locally to martensite. The model presented in this paper is motivated by the work of Peultier et al. [8] and improved by Chemisky [9] and Duval [10]. A brief description of the modeling is given below. For more details, the readers can consult the published work of Chemisky [9] and Duval [10]. The expression of the Gibbs free energy is defined to describe the two key features described above, which are the introduction of pathdependent transformation strain and the description of the twin accommodation mechanisms. Two internal variables are used as well: the volume fraction of martensite $f$ and the average mean strain $\bar{\varepsilon}_{i j}^{T}$ which can be defined by the following equations:

$$
\begin{aligned}
f & =V_{M} / V \\
\bar{\varepsilon}_{i j}^{T} & =\frac{1}{V_{M}} \int_{V_{M}} \varepsilon_{i j}^{T}(r) \mathrm{d} V
\end{aligned}
$$

The transformation strain $E_{i j}^{T}$ can be expressed as follow:

$$
E_{i j}^{T}=f \bar{\varepsilon}_{i j}^{T}
$$

Considering an additive decomposition of strain, the total macroscopic strain $E_{i j}$ is written:

$$
E_{i j}=E_{i j}^{e l}+E_{i j}^{t h}+E_{i j}^{T}
$$

where $E^{e l}$ et $E^{\text {th }}$ are respectively the elastic and thermal strain tensors. The derivation of the phenomenological constitutive models for SMA begins with the choice of a thermodynamic free energy potential. As it is developed in Chemisky et al. [9], the following Gibbs free energy potential variation is defined:

$$
\begin{aligned}
\Delta G= & -\Delta T S^{A}+B\left(T-T_{0}\right) f-\frac{1}{2} \Sigma_{i j} S_{i j k l} \Sigma_{k l}-f \Sigma_{i j} \bar{\varepsilon}_{i j}^{T} \\
& -\Sigma_{i j} \alpha \delta_{i j}\left(T-T_{r e f}\right)+\frac{1}{2} H_{f} f^{2}+\frac{1}{2} H_{\varepsilon} f \bar{\varepsilon}_{i j}^{T} \bar{\varepsilon}_{i j}^{T}
\end{aligned}
$$

where $S^{A}$ is the entropy of the austenite phase, $B=-\Delta S$ is the difference between the entropy of the austenite and the martensite phases, $T$ is the temperature, $H_{f}$ and $H_{\varepsilon}$ are interactions parameters, $\Sigma$ is the macroscopic stress tensor, $S$ is the elastic tensor and $\alpha$ is the thermal expansion coefficient. Thus, driving force variables linked to the internal variables are defined by:

$$
\begin{aligned}
F_{\Sigma_{i j}}= & -\frac{\partial \Delta G}{\partial \Sigma_{i j}}=S_{i j k l} \Sigma_{k l}+\alpha \delta_{i j}\left(T-T_{r e f}\right)+f \bar{\varepsilon}_{i j}^{T}(9) \\
F_{\bar{\varepsilon}_{i j}^{T}}= & -\frac{\partial \Delta G}{\partial \bar{\varepsilon}_{i j}^{T}}=f\left(\Sigma_{i j}^{d e v}-H_{\varepsilon} \bar{\varepsilon}_{i j}^{T}-\lambda_{i j}^{e}\right) \\
F_{f}= & -\frac{\partial \Delta G}{\partial f}=-B\left(T-T_{0}\right)+\Sigma_{i j} \bar{\varepsilon}_{i j}^{T} \\
& -\frac{1}{2} H_{\varepsilon} \bar{\varepsilon}_{i j}^{T} \bar{\varepsilon}_{i j}^{T}-H_{f} f-\lambda_{0}-\lambda_{1}
\end{aligned}
$$




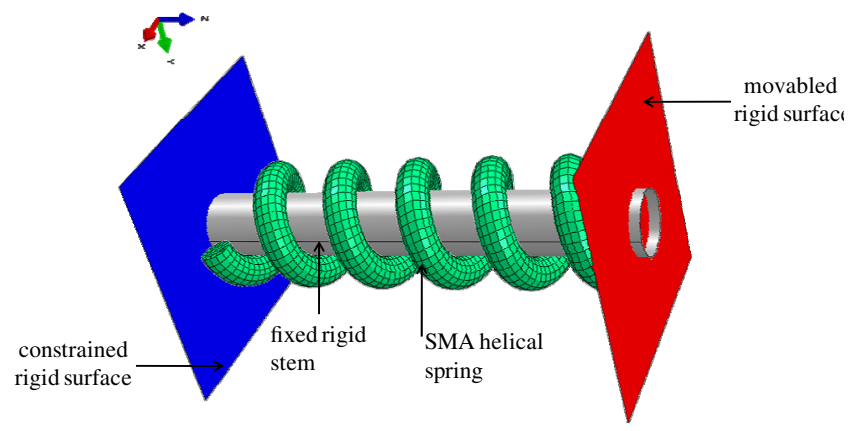

(a)

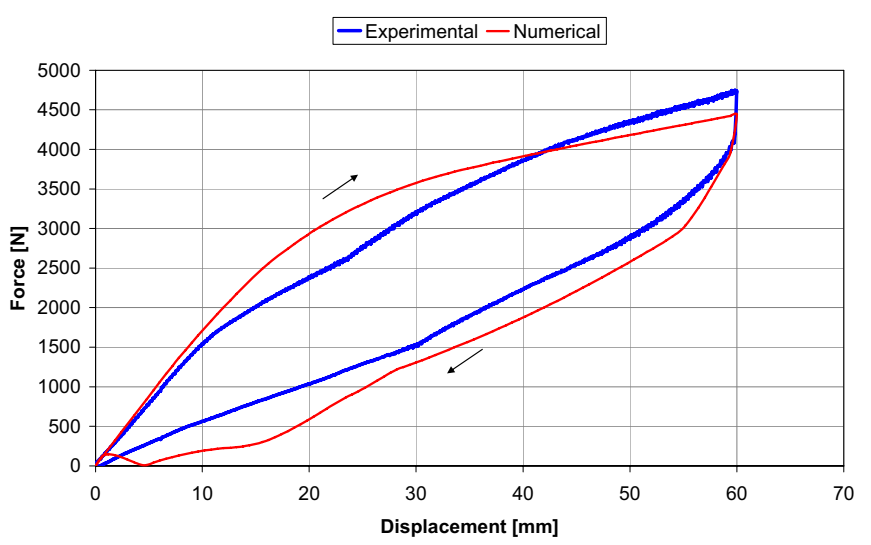

(b)

Fig. 4. Finite element model of the SMA spring in the initial configuration (a) and comparison between the numerical and the experimental investigations (b).

where $\Sigma_{i j}^{d e v}$ is the deviatoric part of the stress tensor. The coefficients $\lambda_{0}, \lambda_{1}$ and $\lambda_{i j}^{\varepsilon}$ are Lagrange multipliers due to the physical limitations. They are defined by the following equations:

and

$$
\lambda_{0}(f)=a_{0} \frac{f-1}{f}, \lambda_{1}(f)=a_{1} \frac{f}{1-f}
$$$$
\lambda_{i j}^{\varepsilon}=\text { function of } \bar{\varepsilon}_{i j}^{T}, \bar{\varepsilon}_{e q}^{T}, \bar{\varepsilon}^{S a t}
$$

\section{Finite element model description and numerical implementation}

The composition of the SMA used in the numerical application is NiTi. Its characteristic phase transformation temperatures measured by electrical resistance evolution are: $M_{F}^{0}=191 \mathrm{~K}, M_{S}^{0}=213 \mathrm{~K}, A_{S}^{0}=205 \mathrm{~K}$ and $A_{F}^{0}=221 \mathrm{~K}$, according to the provider. The material parameters are identified by fitting the experimental curves given in the Figure 2 and previous tensile test of the material given in reference [6], considering a numerical convergence test. The stress distribution is considered converged by using 49731 degrees of freedom.

An appropriate user subroutine (UMAT) is written by $\mathrm{C}$ language in the commercially available finite element program ABAQUS ${ }^{\circledR}$. The numerical implementation is based on 3D finite element approach: the spring is modeled three dimensionally with three dimensional quadratic brick elements with linear interpolation and reduced integration C3D8R (Fig. 4a). Two rigid surfaces in contact with the spring ends are considered. The lower rigid surface is constrained in all directions and a time varying displacement boundary condition is defined for the upper surface for modeling compression. The supporting shaft in the experiments is modeled with a cylindrical rigid surface inside the helical spring, which prevents the spring bending effects during the compression phase. The automatic time increment option in ABAQUS ${ }^{\circledR}$ is used with an initial guess of dividing the loading and unloading steps into 1000 increments and the non-linear geometry option is activated. Finally, the non linear problem is solved by using an implicit scheme with a Newton-Raphson algorithm.

\section{Numerical results}

The load-displacement response for both numerical and experimental cycles is given on Figure $4 \mathrm{~b}$. One can notice the same tendency for both results with a non linear behavior with hysteresis between the compression force and the axial displacement. For a given displacement, the compression force has the same magnitude for the numerical and the experimental results. Consequently, one can affirm that there is a good correlation between the numerical and the experimental results although there had been some minor differences. These differences can be explained by the design of the spring which is not totally perfect and the contact between the rigid plates, the stem and the spring which is difficult to model exactly. This first numerical result shows a good correlation between the experimental and the numerical results. It validates the identification of the material parameters and the implementation of the $3 \mathrm{D}$ model. The displacement magnitude, the von Mises stress and the volume fraction of martensite are respectively shown on Figures $5 \mathrm{a}-5 \mathrm{c}$, for a displacement of the movable plate about $60 \mathrm{~mm}$. One can notice that the transformation phase occurs at this compression level, but no area is totally transformed $\left(f_{\max }=0.64\right)$. Nevertheless, this is sufficient to confer to the spring the typical non linear hysteretic behavior, observed in the experimental part. Now, it is possible to simulate many cycles with different compression ratio, in order to investigate the dynamical behavior of the spring and its damping effect. Finally, it can be said that the numerical simulation proposed in this communication has many similarities with the one 


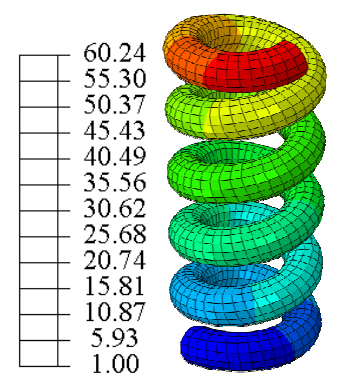

(a) Displacement magnitude $[\mathrm{mm}]$

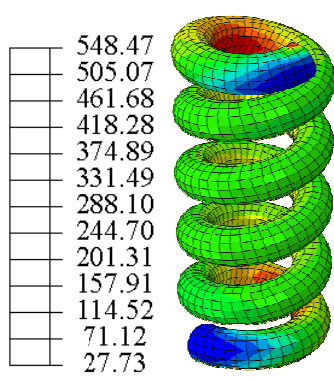

(b) von Mises stress $[\mathrm{MPa}]$

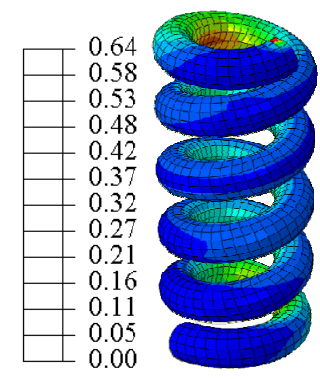

(c) Volume fraction of martensite

Fig. 5. Numerical results at the end of the loading phase (compression displacement about $60 \mathrm{~mm}$ ).

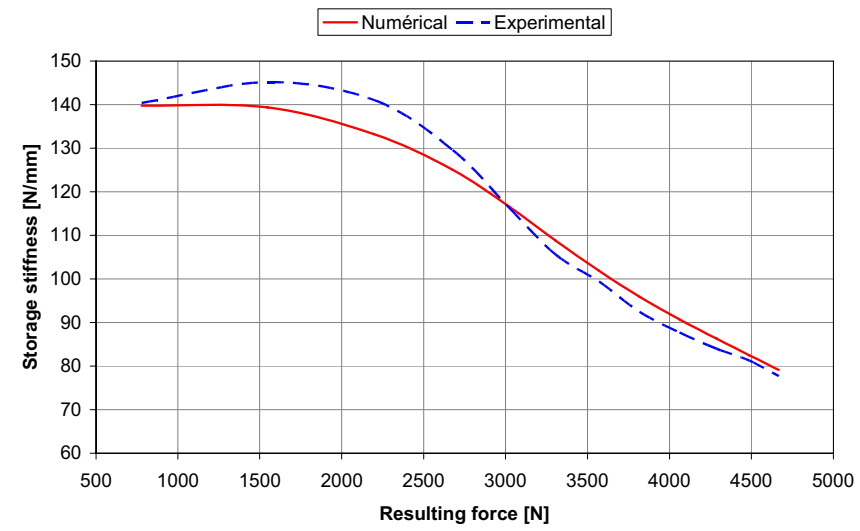

(a)

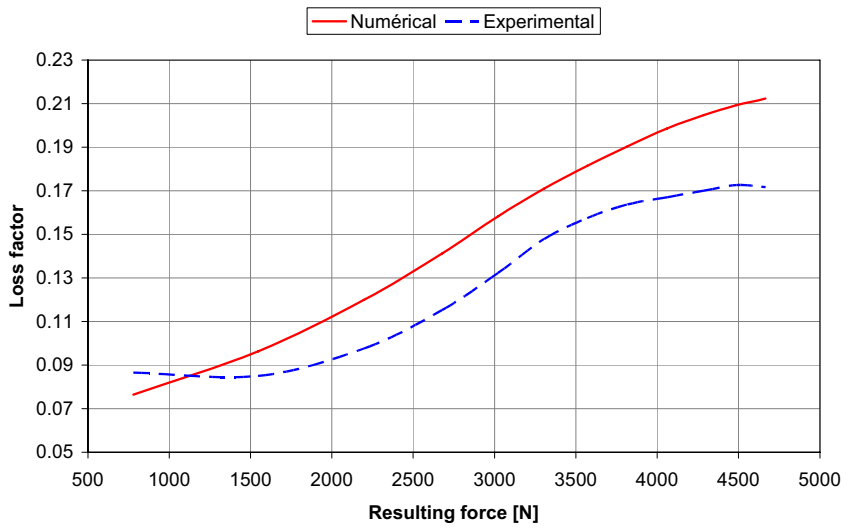

(b)

Fig. 6. Equivalent complex stiffness: storage stiffness (a) and loss factor (b) versus the resulting force.

proposed by Mirzaeifar et al. [7]. The main difference is that the cycles (force-displacement) are used to build the equivalent complex stiffness which is the innovative tool related in this communication in order to investigate the damping effect of SMA structures.

\section{Dynamical behavior and damping effect}

\subsection{Equivalent complex stiffness}

In the mono axial case, the constitutive relation between the resulting compression force $\underline{F}$ and the displacement of the movable rigid surface $\underline{u}$ for a damping material subject to steady state harmonic excitations can be written as:

$$
\underline{F}=\bar{K} \cdot \underline{u} \text { with } \bar{K}=K(1+i \eta)
$$

where $\bar{K}$ is the equivalent complex stiffness, $K$ the storage stiffness and $\eta$ the loss factor. Typically, the loss factor is an image of the damping effect of such a device.

By considering the Valanis endochronic theory [11] and the approximation of the harmonic balance, it is possible to define the storage stiffness and the loss factor. After calculations, their expressions are respectively:

$$
\begin{gathered}
K \sqrt{1+\eta^{2}}=\frac{\omega}{\pi \cdot u_{m}} \\
\times \sqrt{\left(\int_{0}^{T} F(t) \cos (\omega t) d t\right)^{2}+\left(\int_{0}^{T} F(t) \sin (\omega t) d t\right)^{2}} \\
\eta=\frac{\int_{0}^{T} F(t) \cos (\omega t) d t}{\int_{0}^{T} F(t) \sin (\omega t) d t}
\end{gathered}
$$

Computed for each cycle (numerical and experimental ones) with a Matlab ${ }^{(}$subroutine, Figure 6 shows the evolution of the storage stiffness (a) and the loss factor (b) versus the compression force. An increase of the loss factor (thus the damping effect) and a decrease of the storage stiffness is noticed with the resulting compression force. Discrepancies between the numerical and the experimental results are a consequence of discrepancies obtained between the numerical and the experimental load-displacement cycles (Fig. 5). Indeed, the Matlab subroutine used to compute the storage stiffness and the loss factor is the same for both approaches. Thus, if the 


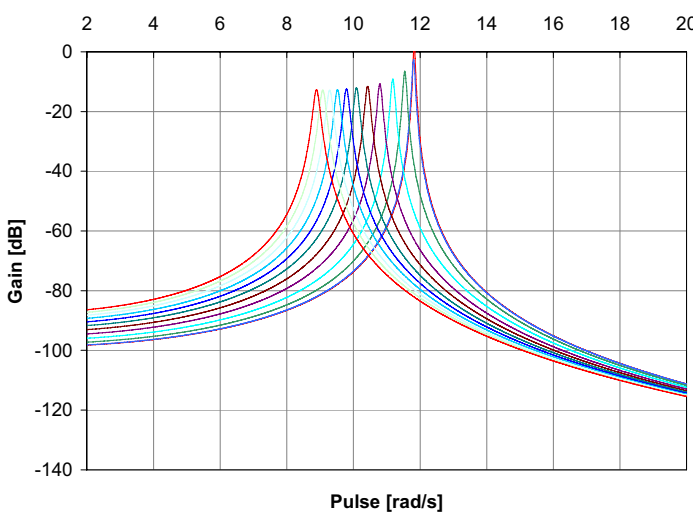

(a)

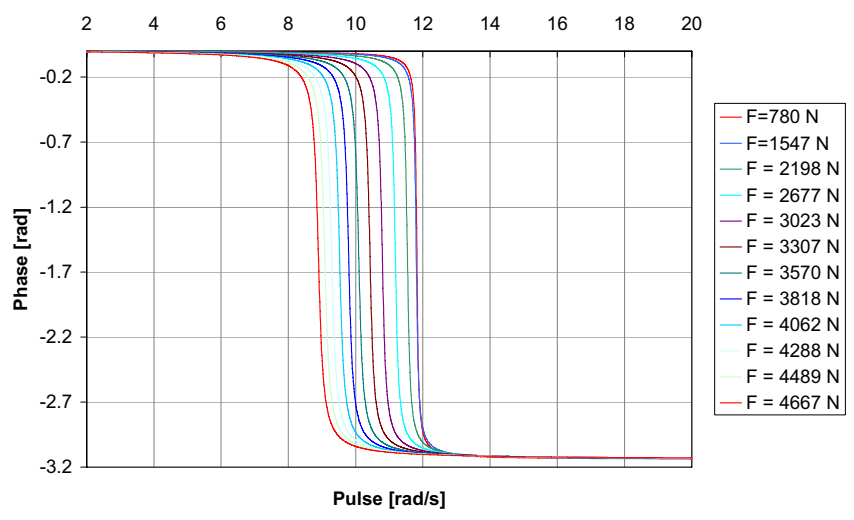

(b)

Fig. 7. Bode diagrams: gain (a) and phase (b).

numerical load-displacement cycle is not totally the same than the experimental one for a given maximal displacement, the storage stiffness and the loss factor will be slightly different. Nevertheless, the same tendency and a good correlation between these results are noticed.

\subsection{Dynamic behavior}

The equivalent complex stiffness is built on the harmonic balance assumption: a Fourier series decomposition of the resulting compression force is done and only its first harmonic is kept. Consequently, it is possible to write the equivalent complex stiffness for non linear material. We precise that the loss factor and the storage stiffness depend on the amplitude displacement for a given cycle. With this definition of the equivalent complex stiffness, the equation (16) is not solved exactly, but the transfer function is written, the real and imaginary parts allow us to build the Bode diagrams which are given on Figure 7. A typical non linear dynamical behavior is noticed with the dependence of the resonance frequency versus the load amplitude.

$$
m \cdot \ddot{u}(t)+k(1+i \eta) \cdot u(t)=F(t)
$$

\section{Conclusion}

In this communication, experimental and numerical compression tests on a SMA helical spring are presented. These investigations lead to investigate the damping effect with the innovative equivalent complex stiffness tool. Consequently, the dynamical behavior of such a device is performed and the non linear dynamical behavior of the spring is noticed. This new numerical modeling tool allows us to improve the non linear modeling of SMA devices in order to develop and optimize applications for control in civil engineering.

\section{References}

[1] F. Gandhi, D. Wolons, Characterisation of the pseudoelastic damping behaviour of shape memory alloy wires using complex modulus, Smart Mater. Struct. 8 (1999) $49-56$

[2] F. Bono, D. Tirelli, Characterisation of materials for the innovative antiseismic techniques, JRC ISPRA, Internal report, 1999

[3] D. Tirelli, V. Renda, F. Bono, Characterisation of shape memory alloys applications to the retrofitting of brick masonry wall by the pseudo-dynamic method and numerical models, JRC ISPRA, Internal report, 2000

[4] M. Collet, E. Foltete, C. Lexcellent, Analysis of the behaviour of a shape memory alloy beam under dynamical loading, Eur. J. Mech. A 20 (2001) 615-630

[5] M.C. Piedboeuf, R. Gauvin, M. Thomas, Damping behavior of shape memory alloys: strain amplitude, frequency and temperature effects, J. Sound Vib. 214 (1998) 895-901

[6] M. Speicher, D. Hodgson, R. Desroches, R. Leon, SMA tension/compression device for seismic retrofit building, JMPEG 18 (2009) 746-753

[7] R. Mirzaeifar, R. Desroches, A. Yavari, A combined analytical, numerical, and experimental study of SMA helical springs, Int. J. Solids Struct. 48 (2010) 611-624

[8] B. Peultier, T. Ben Zineb, E. Patoor, Macroscopic constitutive law of shape memory alloy thermomechanical behaviour, Application to Structure computation (b) FEM, Mech. Mater. 38 (2006) 510-524

[9] Y. Chemisky, A. Duval, E. Patoor, T. Ben Zineb, Constitutive model for shape memory alloys including phase transformation, martensitic reorientation and twins accommodation, Mech. Mater. 43 (2011) 361-376

[10] A. Duval, M. Haboussi, T. Ben Zineb, Modeling of SMA superelastic behavior with non local approach, Phys. Proc. 10 (2010) 33-38

[11] K.C. Valanis, A theory of viscoplasticity without a yield surface, Arch. Mech. 23 (1971) 517-551 